\title{
Efeito da Chuva na Eficiência de Herbicidas Aplicados em Pós-EMERgênCIA SOBRE CORDA-DE-VIOLA ${ }^{1}$
}

\author{
Effect of Rainfall on the Efficiency of Herbicides Applied in Post-Emergence on \\ Ipomoea grandifolia
}

\author{
SOUZA, G.S.F. ${ }^{2}$, MARTINS, D. ${ }^{3}$ e PEREIRA, M.R.R. ${ }^{4}$
}

\begin{abstract}
RESUMO - O objetivo deste trabalho foi avaliar a influência da chuva na eficiência de herbicidas aplicados em pós-emergência em plantas de Ipomoea grandifolia. As plantas de I. grandifolia foram cultivadas em vasos plásticos com capacidade de $2,5 \mathrm{~L}$, em casa de vegetação, com uma planta por vaso. O delineamento experimental utilizado foi o inteiramente casualizado, com quatro repetições, com os tratamentos dispostos em um esquema fatorial $7 \times 8$ (sete tratamentos químicos e oito períodos para ocorrência de chuva após a aplicação dos tratamentos). Os tratamentos químicos constaram da aplicação de glyphosate em cinco formulações comerciais (Roundup Original, Roundup WG, Roundup Transorb, Roundup Transorb R e Roundup Ultra) a 1.080 g e.a. ha-1, amônio-glufosinate (Finale) a 400 g i.a. ha-1 e 2,4-D (DMA 806) a 1.000 g e.a. ha ${ }^{-1}$ e de oito intervalos de tempo para simulação de uma chuva de $15 \mathrm{~mm}$, com duração de cinco minutos: 15', 30', 1h, 2h, 4h, 6h, 8h após a aplicação dos tratamentos e uma testemunha sem chuva, Foram realizadas avaliações visuais de controle das plantas aos 7, 14, 21, 28 e 35 dias após a aplicação e, por ocasião da última avaliação, determinou-se a massa seca das plantas. A ocorrência de chuvas após a aplicação de 2,4-D não alterou a sua eficiência no controle das plantas de I. grandifolia; já os herbicidas amônio-glufosinate e glyphosate, em todas suas formulações testadas, apresentaram redução na eficiência de controle quando da ocorrência de chuvas em até oito horas após a aplicação dos tratamentos.
\end{abstract}

Palavras-chave: amônio-glufosinate, glyphosate, Ipomoea grandifolia, planta daninha, 2,4-D.

\begin{abstract}
The objective of this study was to evaluate the influence of rainfall on the efficiency of herbicides applied in post-emergence on Ipomoea grandifolia plants. I. grandifolia plants were sown in plastic pots with a capacity of $2.5 \mathrm{~L}$ under greenhouse conditions, with only one plant per pot. The experiment was arranged in a completely randomized design, with four replications, with the treatments arranged in a $7 \times 8$ factorial (seven chemical treatments and eight rainfall periods after treatment application). Chemical treatments consisted of application of glyphosate in five commercial formulations (Roundup Original, Roundup WG, Roundup Transorb, Roundup Transorb R, and Roundup Ultra) to 1,080 g a.e. ha-1, glufosinate-ammonium (Finale) to $400 \mathrm{~g}$ a.i. ha $\mathrm{h}^{-1}$ and 2,4-D (DMA 806) and 1,000 g a.e. ha-1, and eight time intervals to simulate $15 \mathrm{~mm}$ rain, lasting five minutes (15, 30', $1 \mathrm{~h}, 2 \mathrm{~h}, 4 \mathrm{~h}, 6 \mathrm{~h}$, and $8 \mathrm{~h}$ ) after application of the treatments and a control without rain. Visual evaluations of plant control were carried out at 7, 14,21, 28, and 35 days after application and at the end of the evaluation period to determine the dry mass of the plants. The occurrence of rain after application of 2, 4-D did not affect their efficiency in controlling $\boldsymbol{I}$. grandifolia plants, whereas the herbicides glufosinate-ammonium and glyphosate, in all the formulations tested, showed reduced efficiency in control upon the occurrence of rainfall up to 8 hours after the treatments.
\end{abstract}

Keywords: Ipomoea grandifolia, weed, glyphosate, 2,4-D, morning glory.

1 Recebido para publicação em 4.8.2011 e aprovado em 25.7.2012.

2 Engo-Agr ${ }^{0}$., M.Sc, Doutorando, Faculdade de Ciências Agronômicas - FCA/UNESP, Caixa Postal 237, 18603-970 Botucatu-SP, <guisasso@hotmail.com>, ${ }^{3}$ Professor Adjunto, Dep. de Produção Vegetal, FCA/UNESP, Botucatu-SP, ${ }^{4}$ Doutora em Agronomia, Pós-Doutoranda, FCA/UNESP, Botucatu-SP. 


\section{INTRODUÇÃO}

As plantas daninhas são um dos mais importantes fatores que afetam a economia agrícola em caráter permanente, pois a sua presença nas culturas agrícolas pode ocasionar diversos prejuízos e o seu controle ainda onera o custeio da cultura. Entre essas espécies que comumente ocorrem nas culturas agrícolas no Brasil, pode-se destacar Ipomoea grandifolia como grande causadora de problemas.

I. grandifolia pertence à família Convolvulaceae, sendo conhecida popularmente como corda-de-viola e apreciada como planta ornamental por apresentar flores vistosas e intensamente coloridas. Entretanto, é uma planta daninha altamente prejudicial em culturas anuais de verão nas regiões Sul, Sudeste e Centro-Oeste do Brasil, por seu ciclo ser maior do que o das culturas e por seus ramos serem muito extensos, interferindo na colheita (Kissman \& Groth, 1999), além de persistir como problema até em áreas com cobertura de palha, como é o caso da cana-deaçúcar no sistema de plantio direto e colheita de cana-de-açúcar crua (Duarte Júnior et al., 2009).

Para eficiente controle dessa espécie e redução dos custos de produção, o uso de herbicidas é uma opção de manejo, principalmente para a dessecação no sistema de plantio direto, com o uso de herbicidas como glyphosate, amônio-glufosinate e 2,4-D. No entanto, a eficácia desses herbicidas está estreitamente relacionada à magnitude do processo de absorção, e vários fatores ambientais podem afetar esse processo, interferindo diretamente na eficácia de ação desses herbicidas, como, por exemplo: temperatura, luminosidade, umidade do solo, umidade relativa do ar, geada e, sobretudo, a ocorrência de precipitações após sua pulverização.

Precipitações pluviais após a aplicação de herbicidas em pós-emergência podem comprometer o controle de plantas daninhas (Behrens \& Elakkad, 1983), principalmente para as operações realizadas em períodos de maior intensidade pluviométrica. Dessa forma, o intervalo de tempo entre a aplicação e a ocorrência de chuvas, a quantidade e a intensidade destas influenciam a eficácia do controle das plantas daninhas (Hammerton, 1967; Anderson \& Arnold, 1984). Além desses fatores, as diferentes formulações e as concentrações dos herbicidas utilizados também podem influenciar na velocidade de absorção e de translocação do ingrediente ativo, assim como na eficiência de controle das espécies de plantas daninhas, como outrora relatado por Bastiani et al. (2000), Martini et al. (2003) e Monquero \& Silva (2007).

Alguns estudos foram realizados para demonstrar o efeito prejudicial da chuva na ação de diversos herbicidas, como os de Jakelaitis et al. (2001), Roman (2001) e Pedrinho Junior et al. (2002), que avaliaram o efeito da chuva simulada na ação de diversas formulações de glyphosate em plantas de Digitaria horizontalis, $B$. decumbens e B. plantaginea, respectivamente.

Anderson et al. (1993) avaliaram os efeitos de uma chuva simulada sobre a eficácia do herbicida amônio-glufosinate em cevada (Hordeum vulgare cv. 'Samson') e capim-rabode-raposa (Setaria viridis). Já Petersen \& Hurle (2001), Oliveira Neto et al. (2010), Mohamad et al. (2010) e Wibawa et al. (2010) também avaliaram a eficácia do amônio-glufosinate no controle de espécies de plantas daninhas, mas sem a ocorrência de chuva após sua aplicação.

Relatos com possíveis efeitos da chuva na eficácia do herbicida 2,4-D são escassos na literatura, porém diversos são os trabalhos que demonstram sua eficiência no controle de inúmeras espécies de plantas daninhas, como os de Mersie \& Parker (1983), Lym (2000), Siebert et al. (2004), Bijanzadeh \& Ghadiri (2006), Everitt \& Keeling (2007), Perim et al. (2009), Caceres et al. (2010), Correa \& Zeitoum (2010) e Cason \& Roost (2011).

Assim, faz-se necessário maior volume de pesquisas para melhor compreensão da interação entre características relacionadas a diferentes espécies de plantas daninhas, aos herbicidas e as condições de tempo. Portanto, objetivou-se avaliar a influência da ocorrência de chuva em diferentes intervalos de tempo após a aplicação, em pós-emergência, dos herbicidas 2,4-D, amônio-glufosinate e glyphosate em diferentes formulações (Roundup Original, Roundup Transorb, Roundup Transorb R, Roundup Ultra e Roundup WG) sobre plantas de I. grandifolia. 


\section{MATERIAL E MÉTODOS}

O estudo foi instalado no mês de janeiro de 2009 no Núcleo de Pesquisas Avançadas em Matologia (NUPAM), pertencente ao Departamento de Produção Vegetal, Setor Agricultura, da Faculdade de Ciências Agronômicas/ UNESP, no município de Botucatu-SP.

Para propiciar condições ideais de crescimento às plantas de I. grandifolia, estas foram semeadas e conduzidas em vasos plásticos com capacidade para $2,5 \mathrm{~L}$ de solo, alocados em casa de vegetação com temperatura controlada em $26{ }^{\circ} \mathrm{C} \pm 2{ }^{\circ} \mathrm{C}$.

O solo utilizado foi adubado com a formulação 04-14-08 de N-P-K e corrigido com calcário dolomítico, de acordo com a análise de solo e recomendação de adubação do Boletim 100 para a cultura do milho. Após a emergência das plântulas, foi feito um desbaste e conduziuse apenas uma planta por vaso.

O delineamento experimental utilizado foi o inteiramente casualizado, com quatro repetições, disposto em um esquema fatorial $7 \mathrm{x} 8$ (sete tratamentos químicos e oito intervalos de chuva); os tratamentos testados constaram da aplicação de glyphosate em cinco formulações comerciais (Roundup Original, Roundup WG, Roundup Transorb, Roundup Transorb R e Roundup Ultra) a 1.080 g e.a. ha ${ }^{-1}$, amônio-glufosinate (Finale) a $400 \mathrm{~g}$ i.a. ha ${ }^{-1} \mathrm{e}$ 2,4-D (DMA 806) a 1.000 g e.a. ha-1.

Foram avaliados oito intervalos de tempo após a pulverização dos herbicidas (15', 30', 1h, $2 \mathrm{~h}, 4 \mathrm{~h}, 6 \mathrm{~h}, 8 \mathrm{~h}$ e sem chuva) para simulação de uma chuva de $15 \mathrm{~mm}$, aplicada durante cinco minutos, simulando uma possivel ocorrência de pancadas de chuva no verão.

Os herbicidas foram aplicados em pósemergência, quando as plantas de corda-deviola apresentavam 25 a $35 \mathrm{~cm}$ de altura (seis a oito folhas), com o uso de um pulverizador costal pressurizado a $\mathrm{CO}_{2}$, equipado com uma barra de pulverização com duas pontas jato plano Teejet XR $110.02 \mathrm{VS}$, distanciadas $50 \mathrm{~cm}$ entre si, com pressão de trabalho de $200 \mathrm{kPa}$, para um consumo de calda de $200 \mathrm{~L} \mathrm{ha}^{-1}$. A simulação da chuva foi feita após a aplicação dos herbicidas, por meio de um pulverizador estacionário, nos tempos estipulados.

Foram realizadas avaliações visuais de controle das plantas aos $7,14,21,28$ e 35 dias após a aplicação (DAA), por meio de uma escala percentual de notas, na qual 0 (zero) correspondeu a nenhuma injúria demonstrada e 100 à morte das plantas, proposta pela Sociedade Brasileira da Ciência das Plantas Daninhas (SBCPD, 1995). Os parâmetros utilizados no estabelecimento das notas para avaliações visuais de controle foram: acúmulo de biomassa, inibição do crescimento, quantidade e uniformidade das injúrias e capacidade de rebrota das plantas.

Ao final do período de avaliação, a massa seca das plantas foi determinada após secagem em uma estufa de ventilação forçada de ar a $65^{\circ} \mathrm{C}$ por 72 horas, com posterior pesagem. Os resultados obtidos para porcentagem de controle e massa seca foram transformados_segundo as equações " $\mathrm{y}=$ $\operatorname{arco} \operatorname{seno}(\operatorname{raiz}(\mathrm{x} / 100)) " \mathrm{e}$ "y = raiz $(\mathrm{x}+1,0)$ ", respectivamente, e submetidos à análise de variância pelo teste $F$, e as médias dos tratamentos, comparadas pelo teste de Tukey $(\mathrm{p}<0,05)$.

\section{RESULTADOS E DISCUSSÃO}

Aos sete dias após a aplicação (DAA) dos herbicidas, já foi possível observar que todos proporcionaram algum efeito tóxico às plantas de corda-de-viola (Tabela 1); entre eles, apenas o amônio-glufosinate apresentou médias de controle da planta daninha consideradas eficientes, com exceção de quando se simulou uma chuva 15 minutos após sua aplicação, pois o controle apresentado neste tratamento pode ser considerado apenas satisfatório. As diferentes formulações de glyphosate mostraram-se ainda ineficientes, independentemente do período de chuva avaliado, com no máximo $28,3 \%$ de controle quando da não ocorrência de chuva com a formulação Roundup Transorb R. Valores semelhantes foram observados por Ramires et al. (2010), que relataram aos 7 DAA um controle de $26,25 \%$ das plantas de corda-de-viola tratadas com 960 g e.a. ha ${ }^{-1}$ de glyphosate. Já o tratamento com o herbicida 2,4-D demonstrou efeito intermediário em relação aos com uso de glyphosate e amônioglufosinate, com médias de controle variando de 23,3 a $50 \%$.

$\mathrm{Na}$ avaliação realizada aos 14 DAA (Tabela 2), verifica-se que todos os tratamentos 
Tabela 1 - Porcentagem de controle de Ipomoea grandifolia aos sete dias após a aplicação de diferentes herbicidas e formulações em intervalos de tempo sem chuva. Botucatu-SP, 2009

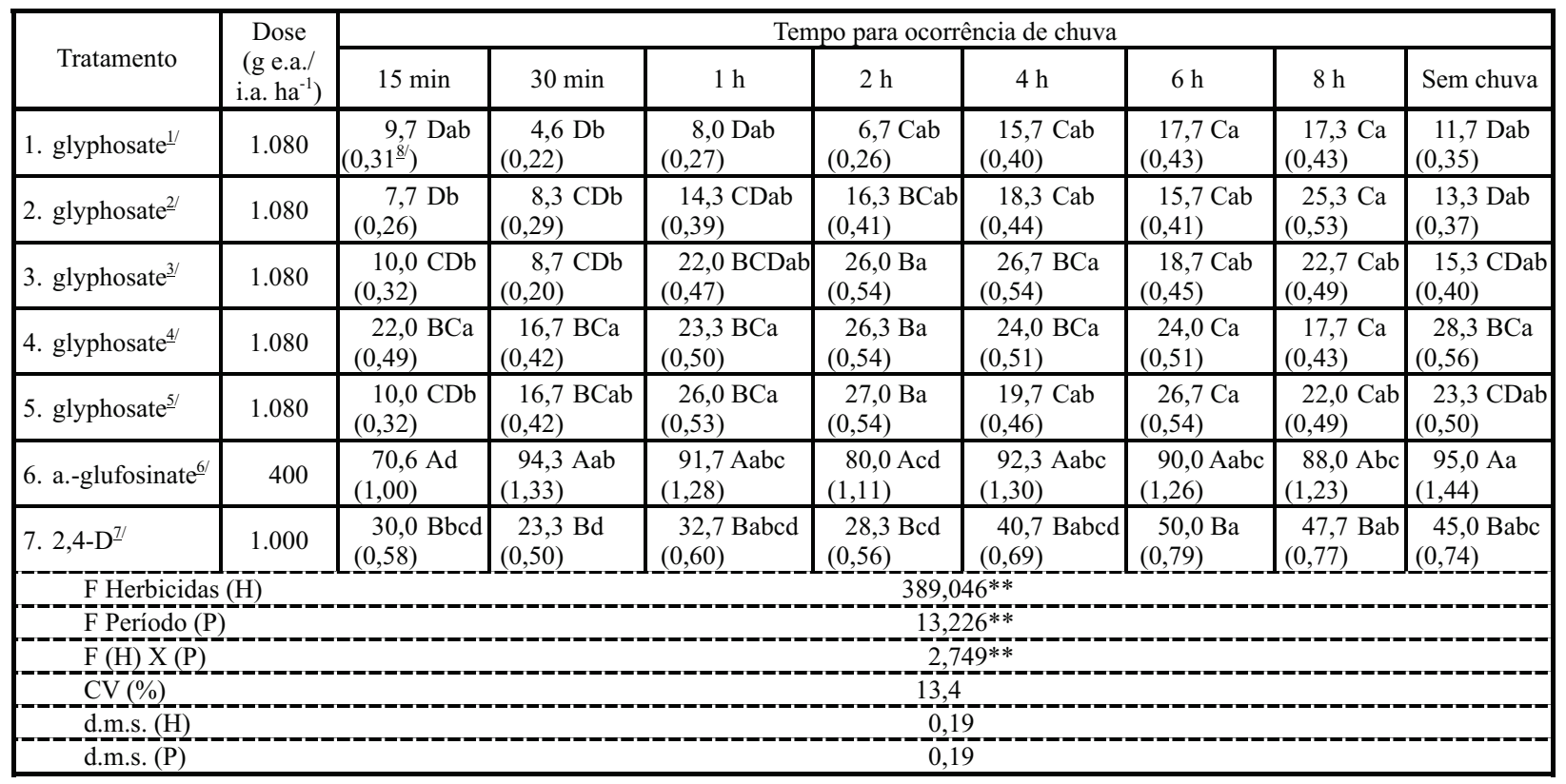

Médias seguidas da mesma letra maiúscula, na coluna, e minúscula, na linha, não diferem estatisticamente entre si pelo teste de Tukey ( $>0,05) .{ }^{* *}$ significativo a $1 \%$ de probabilidade. ${ }^{1 /}$ Roundup Original; ${ }^{2 /}$ Roundup WG; ${ }^{3 /}$ Roundup Transorb; ${ }^{4 /}$ Roundup Transorb R; ${ }^{5 /}$ Roundup Ultra; ${ }^{6 /}$ amônio-glufosinate - Finale; ${ }^{7 /}$ DMA 806. ${ }^{8 /}$ Os dados foram transformados segundo a equação "y $=$ arco seno (raiz $(\mathrm{x} / 100)) "$

Tabela 2 - Porcentagem de controle de Ipomoea grandifolia aos 14 dias após a aplicação de diferentes herbicidas e formulações em intervalos de tempo sem chuva. Botucatu-SP, 2009

\begin{tabular}{|c|c|c|c|c|c|c|c|c|c|}
\hline \multirow{2}{*}{ Tratamento } & \multirow{2}{*}{$\begin{array}{c}\text { Dose } \\
(\mathrm{g} \text { e.a./ } \\
\left.\text { i.a. ha }{ }^{-1}\right)\end{array}$} & \multicolumn{8}{|c|}{ Tempo para ocorrência de chuva } \\
\hline & & $15 \mathrm{~min}$ & $30 \mathrm{~min}$ & $1 \mathrm{~h}$ & $2 \mathrm{~h}$ & $4 \mathrm{~h}$ & $6 \mathrm{~h}$ & $8 \mathrm{~h}$ & Sem chuva \\
\hline 1. glyphosate ${ }^{1 /}$ & 1.080 & \begin{tabular}{|l}
$24,3 \mathrm{Bc}$ \\
$\left(0,51^{\underline{8}}\right)$
\end{tabular} & $\begin{array}{l}19,3 \mathrm{Cc} \\
(0,46)\end{array}$ & $\begin{array}{l}17,3 \mathrm{Cc} \\
(0,43)\end{array}$ & \begin{tabular}{|l}
$24,0 \mathrm{Dc}$ \\
$(0,51)$
\end{tabular} & $\begin{array}{l}28,3 \mathrm{Bc} \\
(0,56)\end{array}$ & $\begin{array}{l}\text { 56,7 CDb } \\
(0,86) \\
\end{array}$ & \begin{tabular}{|c|}
$33,7 \mathrm{Cbc}$ \\
$(0,61)$
\end{tabular} & $\begin{array}{l}96,7 \mathrm{ABa} \\
(1,40)\end{array}$ \\
\hline 2. glyphosate $\mathrm{e}^{2 /}$ & 1.080 & $\begin{array}{l}27,7 \mathrm{Bb} \\
(0,55)\end{array}$ & $\begin{array}{l}21,7 \mathrm{Cb} \\
(0,48)\end{array}$ & $\begin{array}{l}31,7 \mathrm{BCb} \\
(0,59)\end{array}$ & $\begin{array}{l}28,3 \mathrm{CDb} \\
(0,56)\end{array}$ & $\begin{array}{l}40,0 \mathrm{Bb} \\
(0,63)\end{array}$ & $\begin{array}{l}35,7 \mathrm{Db} \\
(0,64)\end{array}$ & $\begin{array}{l}45,0 \mathrm{BCb} \\
(0,73)\end{array}$ & \begin{tabular}{|l}
$89,7 \mathrm{ABa}$ \\
$(1,28)$
\end{tabular} \\
\hline 3. glyphosate ${ }^{3 /}$ & 1.080 & $\begin{array}{l}20,0 \mathrm{Bc} \\
(0,46)\end{array}$ & $\begin{array}{l}24,3 \mathrm{Cc} \\
(0,51)\end{array}$ & $\begin{array}{l}40,7 \mathrm{BCbc} \\
(0,69)\end{array}$ & $\begin{array}{l}47,0 \mathrm{CDbc} \\
(0,76) \\
\end{array}$ & $\begin{array}{l}45,3 \mathrm{Bbc} \\
(0,74)\end{array}$ & $\begin{array}{l}40,0 \mathrm{CDbc} \\
(0,68)\end{array}$ & $\begin{array}{l}65,0 \mathrm{Bb} \\
(0,95) \\
\end{array}$ & $\begin{array}{c}95,3 \mathrm{ABa} \\
(1,39) \\
\end{array}$ \\
\hline 4. glyphosate ${ }^{4 /}$ & 1.080 & $\begin{array}{l}26,0 \mathrm{Bb} \\
(0,53)\end{array}$ & $\begin{array}{l}30,7 \mathrm{BCb} \\
(0,59)\end{array}$ & $\begin{array}{l}46,0 \mathrm{Bb} \\
(0,76)\end{array}$ & $\begin{array}{l}36,7 \mathrm{CDb} \\
(0,66)\end{array}$ & $\begin{array}{l}42,7 \mathrm{Bb} \\
(0,71)\end{array}$ & $\begin{array}{l}43,0 \mathrm{CDb} \\
(0,72)\end{array}$ & $\begin{array}{l}40,0 \mathrm{BCb} \\
(0,68)\end{array}$ & $\begin{array}{c}90,3 \mathrm{ABa} \\
(1,31)\end{array}$ \\
\hline 5. glyphosate ${ }^{5 /}$ & 1.080 & $\begin{array}{l}25,0 \mathrm{Bc} \\
(0,52)\end{array}$ & $\begin{array}{l}43,3 \mathrm{BCbc} \\
(0,73)\end{array}$ & $\begin{array}{l}45,7 \mathrm{Bbc} \\
(0,74)\end{array}$ & $\begin{array}{l}53,3 \mathrm{BCb} \\
(0,82)\end{array}$ & $\begin{array}{l}42,7 \mathrm{Bbc} \\
(0,71)\end{array}$ & $\begin{array}{l}62,7 \text { BCab } \\
(0,93)\end{array}$ & $\begin{array}{l}39,3 \text { BCbc } \\
(0,68)\end{array}$ & $\begin{array}{l}83,3 \mathrm{BCa} \\
(1,15)\end{array}$ \\
\hline 6. a.-glufosinate ${ }^{6 /}$ & 400 & $\begin{array}{l}40,0 \mathrm{Bb} \\
(0,68)\end{array}$ & $\begin{array}{l}98,3 \mathrm{Aa} \\
(1,44)\end{array}$ & $\begin{array}{l}95,0 \mathrm{Aa} \\
(1,35)\end{array}$ & \begin{tabular}{|l}
$84,7 \mathrm{Aa}$ \\
$(1,20)$
\end{tabular} & $\begin{array}{l}90,0 \mathrm{Aa} \\
(1,30)\end{array}$ & $\begin{array}{l}91,7 \mathrm{Aa} \\
(1,29)\end{array}$ & \begin{tabular}{|l}
$91,3 \mathrm{Aa}$ \\
$(1,27)$
\end{tabular} & $\begin{array}{l}98,7 \mathrm{Aa} \\
(1,46)\end{array}$ \\
\hline 7. $2,4-\mathrm{D}^{\mathrm{Z} /}$ & 1.000 & $\begin{array}{l}\text { 68,7 Abc } \\
(0,99)\end{array}$ & $\begin{array}{l}53,7 \mathrm{Bc} \\
(0,82)\end{array}$ & $\begin{array}{l}82,3 \text { Aab } \\
(1,16)\end{array}$ & $\begin{array}{l}\text { 78,7 ABabc } \\
(1,10)\end{array}$ & $\begin{array}{l}\text { 81,3 Aab } \\
(1,14)\end{array}$ & $\begin{array}{l}\text { 85,7 ABab } \\
(1,22)\end{array}$ & $\begin{array}{l}96,0 \mathrm{Aa} \\
(1,38)\end{array}$ & $\begin{array}{l}\text { 69,3 Cbc } \\
(0,99)\end{array}$ \\
\hline \multicolumn{10}{|c|}{ F Herbicidas $(\mathrm{H})$} \\
\hline \multicolumn{10}{|c|}{ F Período $(\mathrm{P})$} \\
\hline \multicolumn{10}{|l|}{$\vec{F}(\mathrm{H}) \mathrm{X}(\mathrm{P})$} \\
\hline \multicolumn{10}{|l|}{ CV $(\%)$} \\
\hline \multicolumn{10}{|l|}{ d.m.s. $(\mathrm{H})$} \\
\hline \multicolumn{10}{|l|}{ d.m.s. (P) } \\
\hline
\end{tabular}

Médias seguidas da mesma letra maiúscula, na coluna, e minúscula, na linha, não diferem estatisticamente entre si pelo teste de Tukey (p $>0,05) .{ }^{* *}$ significativo a $1 \%$ de probabilidade. ${ }^{1 /}$ Roundup Original; $\stackrel{2}{ }$ Roundup WG; $\stackrel{3}{ }$ Roundup Transorb; ${ }^{4 /}$ Roundup Transorb R; $\stackrel{5}{5}$ Roundup Ultra; ${ }^{6 /}$ amônio-glufosinate - Finale; ${ }^{7 /}$ DMA 806. $\stackrel{8}{ }$ Os dados foram transformados segundo a equação "y $=$ arco seno (raiz $(\mathrm{x} / 100))^{\prime \prime}$. 
que não receberam chuva controlaram as plantas de corda-de-viola acima de $83 \%$, a não ser as parcelas tratadas com 2,4-D, que apresentaram média de $69 \%$ de controle. Melhores resultados foram verificados com o uso de 2,4-D quando da ocorrência de chuva 1, 2, 4, 6 e $8 \mathrm{~h}$ após sua aplicação, em comparação à não ocorrência de chuva. Esse fato pode estar relacionado a uma ação conjunta de controle da absorção foliar e radicular, que pode ter ocorrido por um possivel escorrimento da calda até o solo com a aplicação da chuva.

Os valores observados nos tratamentos com aplicação de glyphosate sem a posterior simulação de chuva corroboram os de Zapparoli et al. (2010), que observaram altas taxas de controle de plantas de I. grandifolia submetidas a tratamentos com glyphosate nas formulações Roundup Original, Roundup Transorb e Roundup WG já aos 14DAA, sem a posterior ocorrência de chuvas. Nos demais períodos de simulação de chuva, todas as formulações à base de glyphosate não apresentaram controle satisfatório da planta daninha, sendo todos influenciados negativamente pelo efeito da chuva até essa época de avaliação.

Jakelaitis et al. (2001) também observaram, aos 14 DAA, reduções nas porcentagens de controle de plantas de $D$. horizontalis submetidas à aplicação de glyphosate em várias formulações (CS, WG, Transorb, Zapp e Zapp Qi) quando se simulou uma chuva uma hora após a aplicação dos herbicidas, em relação a períodos maiores de tempo entre a aplicação e a chuva $(2,4,6$ horas e não simulação de chuva).

Os produtos à base de amônio-glufosinate e 2,4-D, ao contrário daqueles à base de glyphosate, apresentaram bom controle das plantas de corda-de-viola, com ocorrência de chuvas a partir de 30 minutos e uma hora após a aplicação, respectivamente, evidenciando assim redução da ação desses dois herbicidas apenas quando da ocorrência de chuva logo após a aplicação deles.

$\mathrm{Na}$ avaliação realizada aos 21 DAA (Tabela 3), nota-se que todos os tratamentos que não receberam simulação de chuva apresentaram controle quase que total das plantas, inclusive o 2,4-D, que na avaliação anterior não apresentava bom controle.
Zapparoli et al. (2010) observaram controle total dessa planta daninha aos 21 DAA com uso das formulações Original, Transorb e WG de glyphosate. Quando se analisa o período de simulação de chuva mais próximo à aplicação dos herbicidas, 15 minutos, todos os tratamentos testados tiveram seu efeito sobre a planta daninha influenciado negativamente pela chuva, a não ser o $2,4-\mathrm{D}$, que apresentou excelente controle, demonstrando assim não ser um produto influenciado pela ocorrência de chuva mesmo após 15 minutos de sua aplicação. Nesta avaliação, ainda foi possível observar que todas as formulações de glyphosate apresentaram acréscimo no controle das plantas em comparação à avaliação anterior, diferentemente do que aconteceu com o amônio-glufosinate, que chegou a apresentar reduções drásticas de controle da planta daninha na maioria dos tratamentos com ocorrência de chuva; isso ocorreu nesse período devido a novas brotações e retomada de crescimento das plantas.

Como registrado na avaliação anterior, aos 28 DAA (Tabela 4), todos os tratamentos que não receberam chuva mantiveram controle excelente da planta daninha. Observa-se que as plantas de I. grandifolia tratadas com 2,4-D, mesmo recebendo chuva em qualquer dos períodos de tempo estudados, foram controladas eficazmente. Siebert et al. (2004) relataram que o 2,4-D também proporcionou excelente controle de uma espécie do mesmo gênero, I. coccinea, mas sem a posterior ocorrência de chuvas.

Já o tratamento com amônio-glufosinate mostrou-se eficiente no controle das plantas de corda-de-viola somente na ausência de chuva. Rodrigues \& Almeida (2005) afirmaram que, para melhor desempenho desse herbicida, é necessário que não ocorra chuva em no mínimo seis horas após sua aplicação.

Todas as formulações à base de glyphosate apresentaram reduções de controle quando houve simulação de chuva, independentemente do tempo para ocorrência desta, em relação ao tratamento sem chuva, sendo o controle ainda menor com a chuva próxima da aplicação dos herbicidas.

Na última avaliação realizada nas plantas de I. grandifolia, aos 35 DAA (Tabela 5), 
Tabela 3 - Porcentagem de controle de Ipomoea grandifolia aos 21 dias após a aplicação de diferentes herbicidas e formulações em intervalos de tempo sem chuva. Botucatu-SP, 2009

\begin{tabular}{|c|c|c|c|c|c|c|c|c|c|}
\hline \multirow{2}{*}{ Tratamento } & \multirow{2}{*}{$\begin{array}{l}\text { Dose } \\
\text { (g e.a./ } \\
\text { i.a. ha-1) }\end{array}$} & \multicolumn{8}{|c|}{ Tempo para ocorrência de chuva } \\
\hline & & $15 \mathrm{~min}$ & $30 \mathrm{~min}$ & $1 \mathrm{~h}$ & $2 \mathrm{~h}$ & $4 \mathrm{~h}$ & $6 \mathrm{~h}$ & $8 \mathrm{~h}$ & Sem chuva \\
\hline 1. glyphosate gl/ $^{1 /}$ & 1.080 & $\begin{array}{r}19,0 \mathrm{Bc} \\
\left(0,45^{\frac{8}{2}}\right)\end{array}$ & $\begin{array}{l}19,3 \mathrm{Bc} \\
(0,45)\end{array}$ & $\begin{array}{l}21,7 \mathrm{Cc} \\
(0,48)\end{array}$ & $\begin{array}{l}31,0 \mathrm{Cbc} \\
(0,59)\end{array}$ & $\begin{array}{l}56,7 \mathrm{Bbc} \\
(0,85)\end{array}$ & $\begin{array}{l}70,3 \mathrm{Bb} \\
(1,00)\end{array}$ & $\begin{array}{l}45,0 \mathrm{Bbc} \\
(0,74)\end{array}$ & $\begin{array}{l}100,0 \mathrm{Aa} \\
(1,57)\end{array}$ \\
\hline 2. glyphosate ${ }^{2 /}$ & 1.080 & $\begin{array}{l}26,7 \mathrm{Bc} \\
(0,54)\end{array}$ & $\begin{array}{l}40,0 \mathrm{Bbc} \\
(0,68)\end{array}$ & $\begin{array}{l}49,0 \text { BCbc } \\
(0,78)\end{array}$ & $\begin{array}{l}51,0 \mathrm{BCbc} \\
(0,80)\end{array}$ & $\begin{array}{l}72,7 \mathrm{Bb} \\
(1,02)\end{array}$ & $\begin{array}{l}69,0 \mathrm{Bb} \\
(0,99)\end{array}$ & $\begin{array}{l}\text { 63,3 Bbc } \\
(0,92)\end{array}$ & $\begin{array}{l}100,0 \mathrm{Aa} \\
(1,57)\end{array}$ \\
\hline 3. glyphosate gl $^{3 /}$ & 1.080 & $\begin{array}{r}31,3 \mathrm{Bd} \\
(0,59)\end{array}$ & $\begin{array}{l}37,0 \mathrm{Bcd} \\
(0,65)\end{array}$ & $\begin{array}{l}56,0 \text { BCbcd } \\
(0,85)\end{array}$ & $\begin{array}{l}56,7 \text { BCbcd } \\
(0,95)\end{array}$ & $\begin{array}{l}72,0 \mathrm{Bbc} \\
(1,01)\end{array}$ & $\begin{array}{l}\text { 67,3 Bbcd } \\
(0,97)\end{array}$ & $\begin{array}{r}78,7 \mathrm{Bb} \\
(1,10)\end{array}$ & $\begin{array}{l}99,7 \mathrm{Aa} \\
(1,54)\end{array}$ \\
\hline 4. glyphosate $e^{4 /}$ & 1.080 & $\begin{array}{l}39,3 \mathrm{Bb} \\
(0,66)\end{array}$ & $\begin{array}{l}53,7 \mathrm{Bb} \\
(0,83)\end{array}$ & $\begin{array}{l}71,7 \mathrm{Bb} \\
(1,02)\end{array}$ & $\begin{array}{l}69,3 \mathrm{ABb} \\
(1,00)\end{array}$ & $\begin{array}{l}70,0 \mathrm{Bb} \\
(1,00)\end{array}$ & $\begin{array}{l}72,7 \mathrm{Bb} \\
(1,02)\end{array}$ & $\begin{array}{l}69,7 \mathrm{Bb} \\
(0,99)\end{array}$ & $\begin{array}{l}99,7 \mathrm{Aa} \\
(1,54)\end{array}$ \\
\hline 5. glyphosate & 1.080 & $\begin{array}{l}35,0 \mathrm{Bb} \\
(0,63)\end{array}$ & $\begin{array}{l}51,7 \mathrm{Bb} \\
(0,81)\end{array}$ & $\begin{array}{l}59,0 \mathrm{BCb} \\
(0,88)\end{array}$ & $\begin{array}{l}71,3 \mathrm{ABb} \\
(1,01)\end{array}$ & $\begin{array}{l}66,7 \mathrm{Bb} \\
(0,96)\end{array}$ & $\begin{array}{l}70,7 \mathrm{Bb} \\
(1,02)\end{array}$ & $\begin{array}{l}42,7 \mathrm{Bb} \\
(0,71)\end{array}$ & $\begin{array}{l}\text { 98,7 Aa } \\
(1,48)\end{array}$ \\
\hline 6. a.-glufosinate ${ }^{6 /}$ & 400 & $\begin{array}{l}10,0 \mathrm{Bd} \\
(0,32)\end{array}$ & $\begin{array}{l}\text { 96,7 Aab } \\
(1,40)\end{array}$ & $\begin{array}{l}\text { 79,7 ABabc } \\
(1,14)\end{array}$ & $\begin{array}{l}53,3 \mathrm{BCc} \\
(0,84)\end{array}$ & $\begin{array}{l}63,3 \mathrm{Bc} \\
(0,94)\end{array}$ & $\begin{array}{l}\text { 69,7 Bbc } \\
(1,05)\end{array}$ & $\begin{array}{l}66,0 \mathrm{Bc} \\
(0,97)\end{array}$ & $\begin{array}{l}99,7 \mathrm{Aa} \\
(1,54) \\
\end{array}$ \\
\hline 7. $2,4-\mathrm{D}^{\mathrm{7} /}$ & 1.000 & $\begin{array}{c}97,0 \mathrm{Aa} \\
(1,44)\end{array}$ & $\begin{array}{r}90,7 \text { Aa } \\
(1,29)\end{array}$ & $\begin{array}{c}98,7 \mathrm{Aa} \\
(1,46)\end{array}$ & $\begin{array}{c}95,0 \mathrm{Aa} \\
(1,37)\end{array}$ & $\begin{array}{r}99,0 \text { Aa } \\
(1,49)\end{array}$ & $\begin{array}{c}99,0 \mathrm{Aa} \\
(1,49)\end{array}$ & $\begin{array}{l}99,3 \mathrm{Aa} \\
(1,50)\end{array}$ & $\begin{array}{l}99,0 \mathrm{Aa} \\
(1,47)\end{array}$ \\
\hline \multicolumn{10}{|c|}{$\overline{\text { F Herbicidas }(\mathrm{H})}$} \\
\hline \multicolumn{10}{|c|}{ F Período $(\mathrm{P})$} \\
\hline \multicolumn{10}{|l|}{$\mathrm{F}(\mathrm{H}) \mathrm{X}(\mathrm{P})$} \\
\hline \multicolumn{10}{|l|}{$\mathrm{CV}(\%)$} \\
\hline \multicolumn{10}{|l|}{ d.m.s. (H) } \\
\hline \multicolumn{10}{|l|}{ d.m.s. (P) } \\
\hline
\end{tabular}

Médias seguidas da mesma letra maiúscula, na coluna, e minúscula, na linha, não diferem estatisticamente entre si pelo teste de Tukey ( $>0,05) .{ }^{* *}$ significativo a $1 \%$ de probabilidade. ${ }^{1 /}$ Roundup Original; $\stackrel{2}{ }$ Roundup WG; $\stackrel{3}{ }$ Roundup Transorb; ${ }^{4 /}$ Roundup Transorb R; 5/ Roundup Ultra; ${ }^{6 /}$ amônio-glufosinate - Finale; ${ }^{7 /}$ DMA 806. ${ }^{8 /}$ Os dados foram transformados segundo a equação "y $=$ arco seno (raiz $(\mathrm{x} / 100)) "$

Tabela 4 - Porcentagem de controle de Ipomoea grandifolia aos 28 dias após a aplicação de diferentes herbicidas e formulações em intervalos de tempo sem chuva. Botucatu-SP, 2009

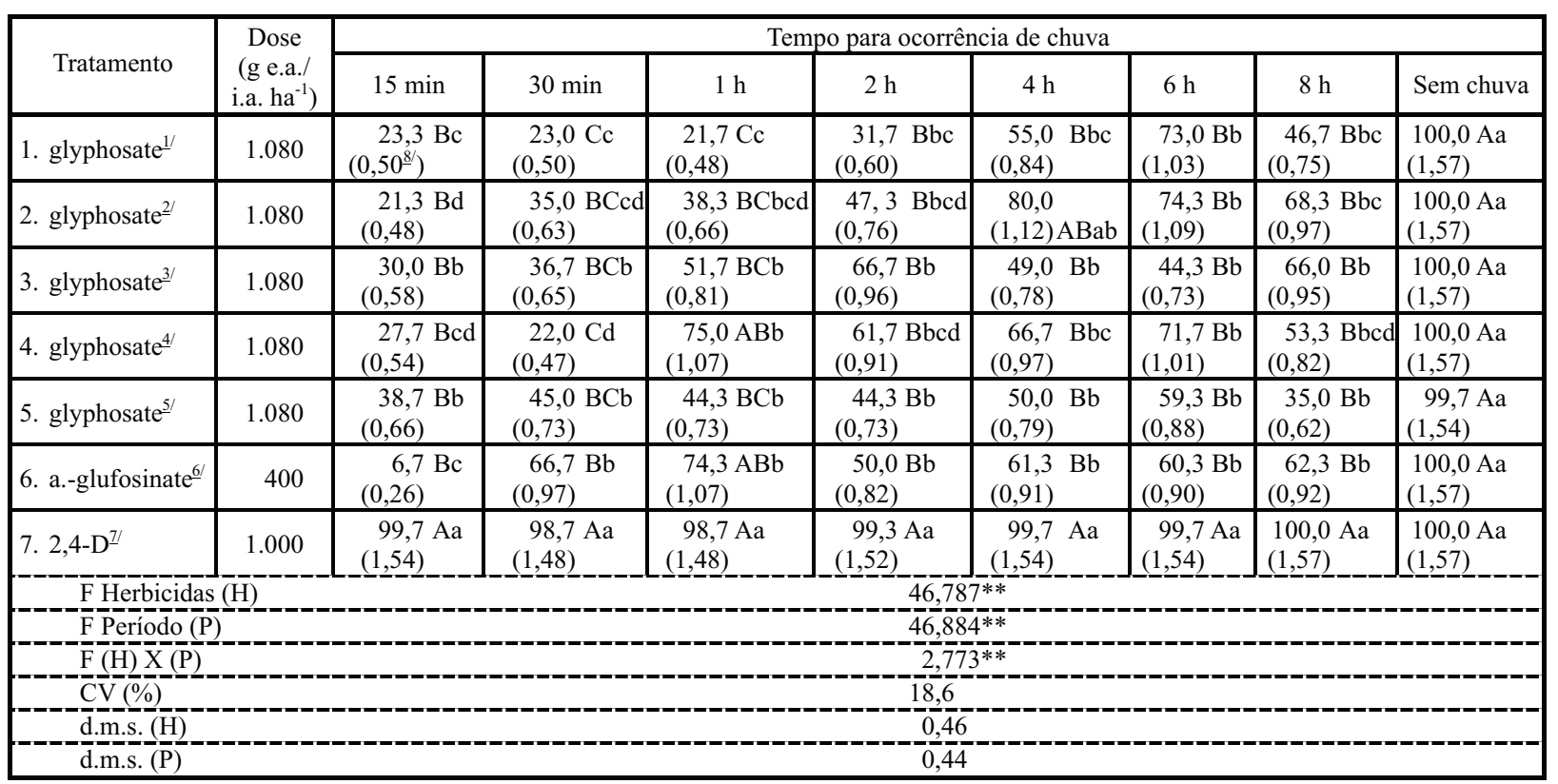

Médias seguidas da mesma letra maiúscula, na coluna, e minúscula, na linha, não diferem estatisticamente entre si pelo teste de Tukey $(\mathrm{p}>0,05) .{ }^{* *}$ significativo a $1 \%$ de probabilidade. ${ }^{1 /}$ Roundup Original; ${ }^{2} /$ Roundup WG; ${ }^{3 /}$ Roundup Transorb; ${ }^{4 /}$ Roundup Transorb R; 5/ Roundup Ultra; ${ }^{6 /}$ amônio-glufosinate - Finale; ${ }^{7 /}$ DMA 806. ${ }^{8 /}$ Os dados foram transformados segundo a equação "y $=$ arco seno (raiz $(\mathrm{x} / 100)) "$ 
Tabela 5 - Porcentagem de controle de Ipomoea grandifolia aos 35 dias após a aplicação de diferentes herbicidas e formulações em intervalos de tempo sem chuva. Botucatu-SP, 2009

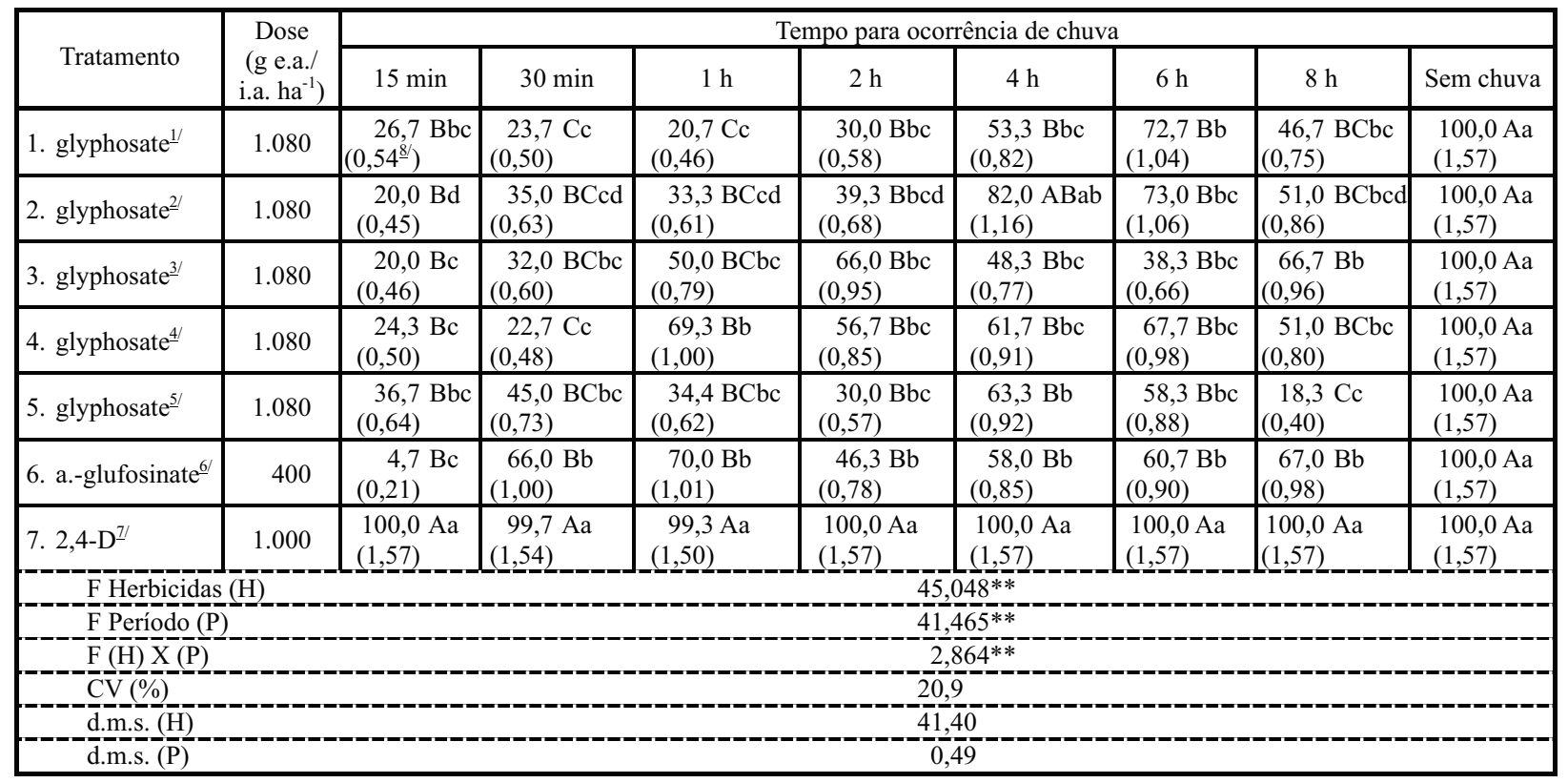

Médias seguidas da mesma letra maiúscula, na coluna, e minúscula, na linha, não diferem estatisticamente entre si pelo teste de Tukey (p>0,05). ** significativo a $1 \%$ de probabilidade. ${ }^{1 /}$ Roundup Original; ${ }^{2 /}$ Roundup WG; ${ }^{3 /}$ Roundup Transorb; ${ }^{4 /}$ Roundup Transorb R; 5/ Roundup Ultra; ${ }^{5 /}$ amônio-glufosinate - Finale; 글 DMA 806. $\stackrel{8}{ } /$ Os dados foram transformados segundo a equação "y $=$ arco seno (raiz $(\mathrm{x} / 100))$ ".

verifica-se que, seguindo os resultados das duas ultimas avaliações, todos os tratamentos testados, quando não receberam simulação de chuva, apresentaram controle máximo da planta daninha. Ramires et al. (2010) também observaram controle quase total dessa espécie aos 35 DAA com o uso do herbicida glyphosate na dosagem de 960 g e.a. ha ${ }^{-1}$.

De acordo com Rodrigues \& Almeida (2005), para diminuir o intervalo temporal entre a aplicação do herbicida e a ocorrência de chuvas, são adicionados surfatantes ao produto, visando conferir maior afinidade da molécula aos lipídios, facilitando assim a absorção foliar. No entanto, todos os tratamentos de chuva afetaram negativamente todas as formulações de glyphosate, fazendo com que nenhuma delas controlasse as plantas de corda-de-viola em nenhum dos períodos de tempo com ocorrência de chuva.

Segundo Boerboon \& Wyse (1988) e MacIsaac et al. (1991), o glyphosate atravessa a cutícula com velocidade moderada, necessitando, em média, de seis horas sem chuvas após a aplicação para haver controle adequado de plantas sensiveis, e é possivel que a absorção relativamente lenta de glyphosate ocorra devido ao valor muito baixo do seu coeficiente de partição octanol por água (4), em comparação ao de outros herbicidas, o que lhe confere baixa lipofilicidade e alta solubilidade em água.

Pedrinho Junior et al. (2002) também notaram acentuada diferença entre formulações de herbicidas inibidores de EPSPs no controle de uma população de plantas daninhas quando se simulou chuva até quatro horas após a aplicação, tendo o glyphosate na formulação Roundup WG $\left(0,5 \mathrm{~kg}\right.$ p.c. ha $\left.{ }^{-1}\right)$ o pior desempenho em relação ao glyphosate nas formulações Roundup SAqC (1 L p.c. ha $\left.{ }^{-1}\right)$, Roundup Transorb (0,75 L p.c. ha $\left.{ }^{-1}\right)$ e Zapp (1,09 L p.c. ha $\left.{ }^{-1}\right)$, não corroborando os dados ora encontrados. Contudo, ressalta-se que as doses utilizadas foram menores que as estudadas neste trabalho.

Todos os periodos de tempo para ocorrência de chuvas após a aplicação do herbicida amônio-glufosinate reduziram sua eficácia de controle em plantas de I. grandifolia para niveis insatisfatórios. Anderson et al. (1993) 
avaliaram os efeitos de uma chuva simulada de 4, 9 e $22 \mathrm{~mm}$ sobre a eficácia do herbicida amônio-glufosinate em cevada (Hordeum vulgare cv. Samson) e capim-rabo-de-raposa (Setaria viridis) e também observaram que as chuvas simuladas logo após a pulverização reduziram a eficácia do herbicida, em ambas as espécies.

Já a eficácia do herbicida 2,4-D não foi alterada por nenhum período de chuva estudado, apresentando excelente controle da planta daninha até mesmo com chuva 15 minutos após sua aplicação. Resultados muito semelhantes foram obtidos por Caceres et al. (2010), que relataram também elevadas taxas de controle para plantas de $I$. nil submetidas ao tratamento com 2,4-D em uma dose muito parecida (1.005 g e.a. ha $\left.{ }^{-1}\right)$, mas sem ocorrência posterior de chuvas.

Observa-se que, corroborando os valores obtidos nas avaliações visuais de controle, em termos biológicos e de maneira geral, a massa seca acumulada nas plantas foi menor quando do tratamento sem chuva (Tabela 6). Todas as formulações de glyphosate foram afetadas pelos diferentes intervalos para ocorrência de chuva - fato esse não observado para o amônio-glufosinate e para o 2,4-D. Contudo, em termos de médias, com exceção do glyphosate na formulação Roundup Original, todos os herbicidas proporcionaram reduções acima de $50 \%$ no acúmulo de matéria seca das plantas de corda-de-viola quando da não ocorrência de chuva em relação à ocorrência 15 minutos após a aplicação deles.

Com base nos resultados, pode-se concluir que a ocorrência de chuvas após a aplicação de 2,4-D não alterou a sua eficiência no controle das plantas de I. grandifolia; já os herbicidas amônio-glufosinate e glyphosate, em todas as suas formulações testadas, apresentaram redução na eficiência de controle quando da ocorrência de chuvas em até oito horas após a aplicação dos tratamentos.

Tabela 6 - Massa seca de plantas (g) de Ipomoea grandifolia aos 35 dias após a aplicação de diferentes herbicidas e formulações em intervalos de tempo sem chuva. Botucatu-SP, 2009

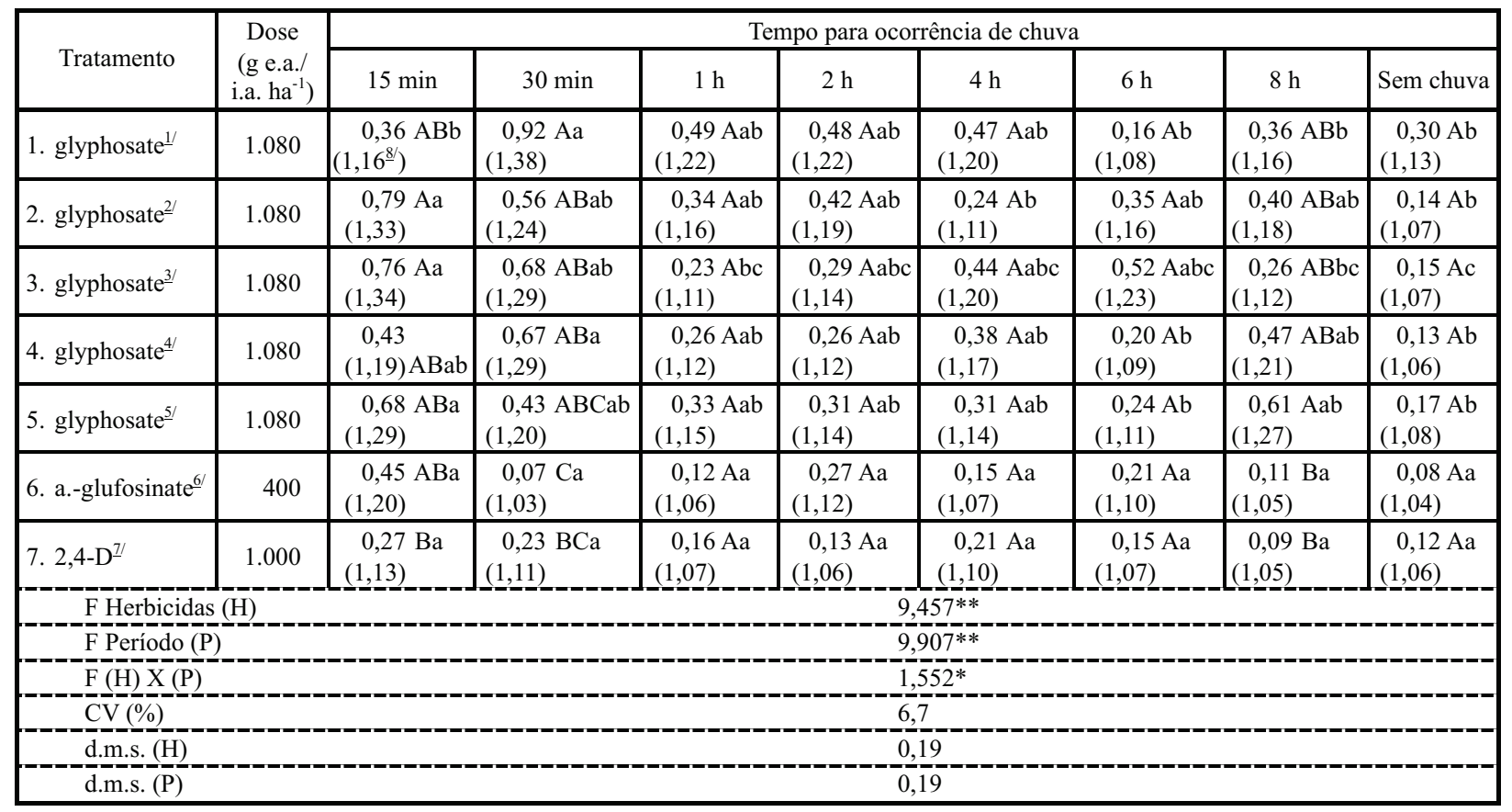

Médias seguidas da mesma letra maiúscula, na coluna, e minúscula, na linha, não diferem estatisticamente entre si pelo teste de Tukey $(\mathrm{p}>0,05) .{ }^{* *}$ significativo a $1 \%$ de probabilidade. ${ }^{1 /}$ Roundup Original; $\stackrel{2}{2}$ Roundup WG; $\stackrel{3}{2}$ Roundup Transorb; ${ }^{4 /}$ Roundup Transorb R; ${ }^{5 /}$ Roundup Ultra; ${ }^{6 /}$ amônio-glufosinate - Finale; ${ }^{7 /}$ DMA 806. $\stackrel{8}{ }$ Os dados foram transformados segundo a equação "y $=$ arco seno (raiz $(\mathrm{x} / 100)) "$ 


\section{LITERATURA CITADA}

ANDERSON, D. M. e al. The influence of soil moisture, simulated rainfall and time of application on the efficacy of glufosinate-ammonium. Weed Res., v. 33, n. 2, p. 149-160, 1993.

ANDERSON, M. D.; ARNOLD, W. E. Weed control in sunflowers (Helianthus annuus) with desmediphan and phenmediphan. Weed Sci., v. 32, n. 3, p. 310-314, 1984

BASTIANI, M. L. R. et al. Influência de chuva simulada após aplicação de herbicida em pós-emergência, sobre o controle de plantas daninhas, em solo com dois níveis de umidade. Planta Daninha, v. 18, n. 1, p. 57-60, 2000

BEHRENS, R.; ELAKKAD, M. A. Influence of rainfall on the phytotoxicity of foliarly applied 2,4-D. Weed Sci., v. 29, n. 3, p. $342-355,1983$.

BIJANZADEH, E.; GHADIRI, H. Effect of separate and combined treatments of herbicides on weed control and corn (Zea mays) yield. Weed Technol., v. 20, n. 3, p. 640-645, 2006.

BOERBOON, C. M.; WYSE, D. L. Influence of glyphosate concentration on glyphosate absorption and translocation in Canada thistle (Cirsium arvense). Weed Sci., v. 36, n. 3, p. 291-295, 1988.

CACERES, N. T. et al. Eficácia do herbicida picloram + 2,4-D no controle de plantas daninhas na cultura da cana-de-açúcar (Saccharum spp.). In: CONGRESSO BRASILEIRO DA CIÊNCIA DAS PLANTAS DANINHAS, 27., 2010, Ribeirão Preto. Anais... Ribeirão Preto: SBCPD, 2010. p. 2298-2302.

CASON, C.; ROOST, B. A. Species selectivity of granular 2,4-D herbicide when used to control eurasian watermilfoil (Myriophyllum spicatum) in Wisconsin Lakes. Invasive Plant Sci. Manag., v.4, n. 2, p. 251-259, 2011

CORREIA, N. M.; ZEITOUM, V. Controle químico de melão-de-são-caetano em área de cana-soca. Bragantia, v. 69, n. 2 , p. $329-337,2010$

DUARTE JÚNIOR, J. B.; COELHO, F. C.; FREITAS, S. P. Dinâmica de populações de plantas daninhas na cana-deaçúcar em sistema de plantio direto e convencional. Semina: Ci. Agr., v. 30, n. 3, p. 595-612, 2009.

EVERITT, J. D.; KEELING, J. W. Weed control and cotton (Gossypium hirsutum) response to preplant applications of dicamba, 2,4-D, and diflufenzopyr plus dicamba. Weed Technol., v. 21, n. 2, p. 506-510, 2007

HAMMERTON, J. L. Environmental factors and susceptibility to herbicides. Weeds, v. 15, n. 4, p. 330-336, 1967.
JAKELAITIS, A. et al. Controle de Digitaria horizontalis pelos herbicidas glyphosate, sulfosate e glyphosate potássico submetidos a diferentes intervalos de chuva após a aplicação.

Planta Daninha, v. 19, n. 2, p. 279-286, 2001.

KISSMANN, K. G.; GROTH, G. Plantas infestantes e nocivas. 2.ed. São Paulo: BASF, 1999. 978 p.

LYM, R. G. Leafy spurge (Euphorbia esula) control with glyphosate plus 2,4-D. J. Range Manag., v. 53, n. 1, p. $68-72,2000$.

MAcISAAC, S. A.; PAUL, R. N.; DEVINE, M. A scanning electron microscope study of glyphosate deposits in relation to foliar uptake. Pestic. Sci., v. 31, n. 1, p. 53-64, 1991.

MARTINI, G.; PEDRINHO JUNIOR, A. F. F.; DURIGAN, J. C. Eficácia do herbicida glifosato-potássico submetido à chuva simulada após a aplicação. Bragantia, v. 62, n. 1, p. $39-45,2003$

MERSIE, W; PARKER, C. Response of teff [Eragrostis tef (Zucc.) Trotter] to 2, 4-D and MCPA at various growth stages. Weed Res., v. 23, n. 1, p. 53-59, 1983.

MOHAMAD, R. B. et al. Management of mixed weeds in young oil-palm plantation with selected broad-spectrum herbicides. J. Trop. Agric. Sci., v. 33, n. 2, p. 193-203, 2010.

MONQUERO, P. A.; SILVA, A. C. Efeito do período de chuva no controle de Euphorbia heterophylla e Ipomoea purpurea pelos herbicidas glyphosate e sulfosate. Planta Daninha, v. 25, n. 2, p. 399-404, 2007.

OLIVEIRA NETO, A. M. et al. Manejo de Conyza bonariensis com glyphosate +2,4-D e amônio-glufosinate em função do estádio de desenvolvimento. R. Bras. Herbic., v. 9, n. 3 , p. $73-80,2010$

PEDRINHO JUNIOR, A. F. F. et al. Momento da chuva após a aplicação e a eficácia dos herbicidas sulfosate e glyphosate aplicados em diferentes formulações. Planta Daninha, v. 20, n. 1, p. $115-123,2002$.

PERIM, L. et al. Eficácia do herbicida amicarbazone no controle em pós-emergência de espécies de corda-de-viola (Ipomoea grandifolia e Merremia cissoids). R. Bras. Herbic. v. 8, n. 1, p. $19-26,2009$

PETERSEN, J.; HURLE, K. Influence of climatic conditions and plant physiology on glufosinate-ammonium efficacy Weed Res., v. 41, n. 1, p. 31-39, 2001.

RAMIRES, A. C. et al. Controle de Euphorbia heterophylla e Ipomoea grandifolia com a utilização de glyphosate isolado ou em associação com latifolicidas. Planta Daninha, v. 28, n. 3, p. 621-629, 2010.

RODRIGUES, B. N.; ALMEIDA, F. S. Guia de herbicidas. 5.ed. Londrina: IAPAR, 2005. 591 p. 
ROMAN, E. S. Influência de chuva simulada na eficácia de diferentes formulações e doses de glyphosate. R. Bras. Herbic., v. 2, n. 1, p. 119-124, 2001.

SIEBERT, J. D.; GRIFFIN, J. L.; JONES, C. A. Red morningglory (Ipomoea coccinea) Control with 2,4-D and Alternative herbicides. Weed Technol., v. 18, n. 1, p. 38-44, 2004.

SOCIEDADE BRASILEIRA DA CIÊNCIA DAS PLANTAS DANINHAS - SBCPD. Procedimentos para instalação, avaliação e análise de experimentos com herbicidas. Londrina: SBCPD, 1995. 42 p.
WIBAWA, W. et al. Efficacy and cost-effectiveness of three broad-spectrum herbicides to control weeds in immature oil palm plantation. J. Trop. Agric. Sci., v. 33, n. 2, p. 233-241, 2010 .

ZAPPAROLI, R. A. et al. Comportamento de três formulações de glyphosate no controle da espécie Ipomoea grandifolia em condições controladas. In: CONGRESSO BRASILEIRO DA CIÊNCIA DAS PLANTAS DANINHAS, 27., 2010, Ribeirão Preto. Anais... Ribeirão Preto: SBCPD, 2010. p. $1102-1105$ 